\title{
Violations of local duality in the heavy quark sector
}

\author{
P. Colangelo a, C.A. Dominguez ${ }^{\text {b }}$, G. Nardulli ${ }^{a, c}$ \\ a Istituto Nazionale di Fisica Nucleare, Sezione di Bari, Italy \\ ${ }^{b}$ Institute of Theoretical Physics and Astrophysics, University of Cape Town, Rondebosch 7700, South Africa \\ c Dipartimento di Fisica, Universitá di Bari, Italy \\ Received 30 May 1997 \\ Editor: R. Gatto
}

\begin{abstract}
We examine the origin of possible failures of the quark-hadron local duality. In particular, we consider a correlator of two currents comprising heavy quark operators, and compare the evaluation by the Operator Product Expansion with the result obtained inserting an infinite set of hadronic states, in the $N_{c} \rightarrow \infty$ limit. Whereas the smeared spectral functions agree with each other, their local behaviour is different. The difference manifests itself in the form of a term $\mathcal{O}(\Lambda / \epsilon)(\epsilon$ being the residual energy) that is not present in the expansion in powers of $\Lambda / \epsilon$ predicted by the OPE. (C) 1997 Elsevier Science B.V.
\end{abstract}

\section{Introduction}

Quark-gluon/hadron duality represents a key concept in the theoretical description of inclusive hadronic processes. By its use, high energy processes such as, e.g., $e^{+} e^{-}$annihilation, can be computed in terms of hadronic matrix clements of operators in an expansion, the Operator Product Expansion (OPE), whose leading term is represented by the perturbative QuantumChromo-Dynamics expression. As a matter of fact, duality provides the tool to extrapolate from the deep Euclidean region, where the OPE is defined, to the Minkowski domain, where physical observables are measured. The basic assumption underlying this tradeoff between hadronic quantities and quark-gluon amplitudes is that, at high energy and/or momentum transfer, the hadronic behaviour should be well described by quark and gluon interactions, provided that sufficiently inclusive variables are considered.

In the last few years this approach has been ex- tended to the decays of hadrons containing one heavy quark, exploiting the presence in these systems of a large parameter, the heavy quark mass $m_{Q}$, that can be taken, at least formally, infinitely large: $m_{Q} \rightarrow \infty[1]$. In this extension, however, a clear distinction must be maintained between semileptonic and nonleptonic decays. As a matter of fact, in gencral, the hadronic and the OPE amplitudes cannot be identical even at very high momentum transfer, due to the different structure of their singularities: multiparticle thresholds in the former case and quark-gluon production thresholds in the latter. For semileptonic heavy hadron decays, however, this difficulty does not prevent duality to hold; as a matter of fact, in computing the semileptonic inclusive width, one has to integrate over lepton variables, which amounts to a smearing of the OPE width. The equality between smeared OPE and physical, i.e. hadronic, widths is sometimes referred to as global duality (assumed to be distinct from local duality, i.e. without smearing) and it is generally be- 
lieved that global duality holds between quark-gluon and hadronic cross sections [2]; for $B$ and $\Lambda_{b}$ decays it has been proved explicitly in [3] to two orders in the $1 / m_{Q}$ expansion and to the first order in $\alpha_{s}$, in the so-called small-velocity (SV) limit $\left(m_{b}, m_{c} \gg\right.$ $m_{b}-m_{c} \gg \Lambda_{\mathrm{QCD}}$ ) [4].

For nonleptonic heavy hadron decays, on the other hand, there are no lepton momenta to be integrated and, therefore, one cannot use global duality to prove the identification of OPE and hadronic observables. Therefore local duality must be assumed, which is a stronger hypothesis. For this reason, the validity of OPE for the computation of nonleptonic heavy hadron inclusive decays appears more debatable. For example in [5] it has been argued that the discrepancy between the OPE prediction $\tau\left(\Lambda_{b}\right) / \tau\left(B_{d}\right)>0.9^{1}$ and the experimental result $\tau\left(\Lambda_{b}\right) / \tau\left(B_{d}\right)=0.78 \pm 0.04$ [9] might be solved assuming a violation of local duality with the appearance of a $\mathcal{O}\left(1 / m_{Q}\right)$ correction not predicted by OPE. In this context, it may be useful to observe that in [10] local duality has been proved for nonleptonic $\Lambda_{b}$ and $B$ inclusive decays, for the first two terms in the $1 / m_{b}$ expansion, and at the order $\alpha_{s}$ in the perturbative expansion, assuming factorization of the weak amplitudes. Also this result, however, holds in the SV limit, i.e. in a kinematical regime which is far off from the physical one; therefore, in absence of a general proof, the failure of local duality, with the appearance of $\mathcal{O}\left(1 / m_{Q}\right)$ corrections to OPE, remains a logical possibility and constitutes a simple explanation of the $\Lambda_{b}$ lifetime data.

It would be clearly extremely important to understand, in this scenario, the origin of this possible $1 / m_{Q}$ correction to the Operator Product Expansion, but this appears, at the moment, a formidable task since, at the present stage of (analytical) understanding of nonper turbative QCD, no first-principle answer can be given to the question of how good duality really is and which violations it may suffer. A more modest, but, nevertheless, potentially instructive aim could be to prove the existence of a violation of local duality and the emergence of a $1 / m_{Q}$ correction in some definite model. This is the main purpose of the present letter.

There have been some other recent studies on the validity of the quark-gluon/hadron duality in connection with nonperturbative QCD applications in the heavy

\footnotetext{
${ }^{1}$ For a discussion see $[6-8]$.
}

quark sector $[11,12]$. Also these works are motivated by the hope to improve the precision with which various observables are determined using the OPE coupled with duality. For example, the model studied in [11] is based on an Ansatz for the evaluation of the twopoint function involving the difference between scalar and pseudoscalar heavy-light currents. In the chiral limit (for the light flavour) and the infinite mass limit (for the heavy flavour), the coefficients of the OPE for this correlator can be calculated analytically. A spectral function model (in the time-like region) is then expanded in a power series (in the space-like region) and compared with the exact result. From this comparison one can gauge the validity of (local) duality. In this letter we consider the same two-point function and we analyze two models: the first model, to be studied in the next Section, represents a slight modification of [11] and leads to a maximal violation of duality. The second model, discussed in Section 3, uses results for the hadronic evaluation of the two-point function obtained by a constituent quark approach; therefore it represents an improvement as compared to the previous Ansätze that are ad hoc assumptions without physical justification. This calculation explicitly shows a violation of local duality in the form of an unexpected $\mathcal{O}\left(1 / m_{Q}\right)$ corrcction to OPE.

\section{Mathematical models for the two-point function}

We begin by considering the following two-point function:

$$
\begin{aligned}
& \Pi(q)=\frac{i}{4} \int d x e^{i q x} \\
& \quad \times\left\langle 0\left|T\left(J_{S}(x) J_{S}^{\dagger}(0)\right)-T\left(J_{P}(x) J_{P}^{\dagger}(0)\right)\right| 0\right\rangle \\
& =\Pi_{S}(q)-\Pi_{P}(q),
\end{aligned}
$$

where the scalar $\left(J_{S}\right)$ and the pseudoscalar $\left(J_{P}\right)$ currents are

$$
\begin{aligned}
& J_{S}(x)=\bar{Q}(x) q(x), \\
& J_{P}(x)=\bar{Q}(x) i \gamma_{5} q(x),
\end{aligned}
$$

and $Q(x), q(x)$ are heavy and light quark operators, respectively. This two-point function is particularly simple, as in the chiral limit $m_{q} \rightarrow 0$ it vanishes in 
perturbation theory. Hence, $\Pi(q)$ is entirely nonperturbative. Additional simplifications take place in the infinite mass limit $m_{Q} \rightarrow \infty$, where it is convenient to write

$q^{\mu}=\left(m_{Q}-\epsilon, \mathbf{q}=0\right)$.

In this limit the correlator becomes a function of $\epsilon$, i.e.

$\Pi(\epsilon)=\frac{1}{4} \int_{0}^{1 \infty} d \tau e^{-\epsilon \tau} \Phi(\tau) \quad(\epsilon>0)$,

where $\Phi(\tau)$ is sometimes called the nonlocal quark condensate [13]

$\Phi\left(\sqrt{-x^{2}}\right)=\left\langle 0\left|\bar{q}(x) e^{i g_{s} \int_{0}^{x} d y^{\mu} A_{\mu}(y)} q(0)\right| 0\right\rangle$.

In the limit $\epsilon \gg \Lambda_{\mathrm{QCD}}$, the OPE expression for $\Pi(\epsilon)$ is given by

$$
\begin{aligned}
& \Pi_{\mathrm{OPE}}(\epsilon)=\frac{\langle 0|\bar{q} q| 0\rangle}{4 \epsilon} \\
& \quad \times\left[1-\frac{m_{0}^{2}}{8 \epsilon^{2}}+c_{4} \frac{m_{0}^{4}}{\epsilon^{4}}-c_{6} \frac{m_{0}^{6}}{\epsilon^{6}}+\ldots\right],
\end{aligned}
$$

where $\langle 0|\bar{q} q| 0\rangle=(-240 \mathrm{MeV})^{3}$,

$m_{0}^{2}=\frac{\left\langle 0\left|g_{s} \bar{q} \sigma_{\mu \nu} G^{\mu \nu} q\right| 0\right\rangle}{\langle 0|\bar{q} q| 0\rangle}=0.8 \pm 0.2 \mathrm{GeV}^{2}$

The positive coefficients $c_{2 n}$ above, depend on the actual form of the nonlocal condensate. We note explicitly the alternating signs in Eq. (7) and the absence of even powers of $\epsilon^{-1}$; both these features are consequences of the general principles on which the OPE is based [11]. In particular, the absence of a $D=4$ term, proportional to the gluon condensate $\left\langle\alpha_{s} G^{2}\right\rangle$, is due to the limit $m_{Q} \rightarrow \infty, m_{q} \rightarrow 0$.

Let us now briefly review the model proposed in [11] to analyze duality violations. It is given by

$\Pi(\epsilon)=\frac{\langle 0|\bar{q} q| 0\rangle}{4 \bar{\Lambda}} \beta\left(\frac{\epsilon+\bar{\Lambda}}{2 \bar{\Lambda}}\right)$,

where $\bar{\Lambda}$ is a parameter and

$\beta(z)=\frac{1}{2}\left[\psi\left(\frac{z+1}{2}\right)-\psi\left(\frac{z}{2}\right)\right]$, $\psi(z)$ being the logarithmic derivative of the Gamma function. The model correlator admits the following series expansion

$$
\Pi(\epsilon)=\frac{\langle 0|\bar{q} q| 0\rangle}{2 \bar{\Lambda}} \sum_{j=0}^{\infty} \frac{(-1)^{j}}{\lambda \epsilon+2 j+1},
$$

where $\lambda=1 / \bar{\Lambda}$. From this expression for $\Pi(\epsilon)$ we get, by the Mellin transform,

$$
\Phi(\tau)=\frac{\langle 0|\bar{q} q| 0\rangle}{\cosh (\bar{\Lambda} \tau)} .
$$

For $\epsilon \rightarrow \infty, \Pi(\epsilon)$ has the asymptotic expansion

$$
\Pi(\epsilon)_{\epsilon \rightarrow \infty} \frac{\langle 0|\bar{q} q| 0\rangle}{4 \epsilon} \sum_{n=0}^{\infty} E_{2 n} \frac{\bar{\Lambda}^{2 n}}{\epsilon^{2 n}}
$$

where $E_{2 n}$ are Euler numbers $\left(E_{0}=1, E_{2}=-1, E_{4}=\right.$ $\left.5, E_{6}=-61, \ldots\right)$ [14].

Comparison of this result with that of the OPE, Eq. (7), indicates that this model is able to reproduce the right power structure of $\Pi(\epsilon)$. The associated spectral density corresponds to an infinite number of equally spaced poles located along the negative $\epsilon$ axis, these poles having residues alternating in sign. After smearing, the spectral function looks rather reasonable [11]. However it is easy to show that this result strongly depends on the Ansatz (8). As a matter of fact, let us consider the slightly modified model

$$
\Pi(\epsilon)=\text { const. } \times \sum_{j=1}^{\infty} \frac{(-1)^{j}}{\lambda \epsilon+2 j}
$$

instead of (10). This implies

$$
\Phi(\tau)=\text { const. } \times\left[\tanh \left(\frac{\tau}{\lambda}\right)-1\right] .
$$

In the limit $\epsilon \rightarrow \infty$, this $\Pi(\epsilon)$ becomes

$\Pi(\epsilon)_{\epsilon \rightarrow \infty}$ const. $\times \frac{1}{\epsilon}\left[1+\sum_{n=0}^{\infty} \frac{C_{2 n+1}}{\epsilon^{2 n+1}}\right]$.

A comparison with the OPE result, Eq. (7), shows that except for the first term, the power structure is wrong, so that one obtains a maximal violation of duality. This model is still quite realistic. The spectral function is also made of an infinite number of zerowidth resonances, with alternating sign residues, and 
located along the negative $\epsilon$ axis. The only difference with the model of [11] is a shift in the location of the poles, due to the absence of the unit factor in the denominator of Eq. (13).

From this example we learn that the agreement found in [11] between the hadronic evaluation of the correlator and the OPE result might be fortuitous; in order to get a deeper understanding we compute now the correlator Eq. (1) using a more realistic model for the spectral density.

\section{A realistic model for the hadronic correlator}

We evaluate the two-point function (1) by inserting hadronic states between the currents. In general this can only be done in some approximation: we choose to insert an infinite number of states, but in the $N_{c} \rightarrow$ $\infty$ limit ( $N_{c}=$ number of colours), where the surviving contributions are $J^{P}=0^{+}$and $0^{-}$single particle states, contributing respectively to the scalar $\left(\Pi_{S}\right)$ and pseudoscalar $\left(\Pi_{P}\right)$ part of $\Pi$. By denoting $\left|S_{n}\right\rangle$ and $\left|P_{n}\right\rangle$ these states, with masses $M_{S_{n}}$ and $M_{P_{n}}$ respectively, we define the current-particle matrix-elements:

$$
\begin{aligned}
& \left\langle 0\left|J_{S}\right| S_{n}\right\rangle=\frac{M_{S_{n}}^{2}}{m_{Q}} f_{S_{n}}, \\
& \left\langle 0\left|J_{P}\right| P_{n}\right\rangle=\frac{M_{P_{n}}^{2}}{m_{Q}} f_{P_{n}} .
\end{aligned}
$$

In the $m_{Q} \rightarrow \infty$ limit we have

$$
\begin{aligned}
& M_{S_{n}}=m_{Q}+\delta_{S_{n}}+\mathcal{O}\left(\frac{1}{m_{Q}}\right), \\
& M_{P_{n}}=m_{Q}+\delta_{P_{n}}+\mathcal{O}\left(\frac{1}{m_{Q}}\right) .
\end{aligned}
$$

The binding energies $\delta_{S_{n}}$ and $\delta_{P_{n}}$ can be obtained by solving the wave equation

$$
\begin{aligned}
& {\left[\sqrt{-\nabla^{2}+m_{Q}^{2}}+\sqrt{-\nabla^{2}+m_{q}^{2}}+V(\boldsymbol{r})\right] \Psi_{n}(\boldsymbol{r})} \\
& \quad=M_{n} \Psi_{n}(\boldsymbol{r})
\end{aligned}
$$

in the limit $m_{Q} \rightarrow \infty, m_{q} \rightarrow 0$. Assuming a central potential $V(\boldsymbol{r})=V(r)$ we can write $\Psi_{n}(\boldsymbol{r})=$ $Y_{\ell m}(\hat{r}) \frac{u_{\rho}^{(n)}(r)}{r}$. The scalar particles are obtained for $\ell=$ 1 ( $P$-waves) and the pseudoscalar particles for $\ell=0$
( $S$-waves). The corresponding equation [15] for $\delta_{S_{u}}$, $\delta_{P_{n}}$ is given, in this limit, by

$$
\begin{aligned}
& {\left[V(r)-\delta_{\ell}^{(n)}\right] u_{\ell}^{(n)}(r)} \\
& \quad+\frac{2}{\pi} \int_{0}^{\infty} d r^{\prime} \int_{0}^{\infty} d k k_{\ell}(k r) \chi_{\ell}\left(k r^{\prime}\right) u_{\ell}^{(n)}\left(r^{\prime}\right) \\
& \quad=0
\end{aligned}
$$

where the relation between $\delta_{S_{n}}, \delta_{P_{n}}$ in (18), (19) and $\delta_{\ell}^{(n)}$ is as follows: $\delta_{0}^{(n)}=\delta_{P_{n}}, \delta_{1}^{(n)}=\delta_{S_{n}}$; moreover, $\chi_{\ell}(x)=x j_{\ell}(x) \quad\left(j_{\ell}\right.$ are the spherical Bessel functions).

$V(r)$ is the static interquark potential; several forms have been studied in the literature which reproduce the experimental spectrum of the heavy $Q \vec{q}$ mesons. In general they behave linearly $V(r) \simeq \mu^{2} r$ for $r \rightarrow$ $\infty$ and have a coulombic behaviour $\left(V(r) \simeq \frac{\alpha_{s}}{r}\right)$ at small distances. In order to simplify our discussion we assume

$V(r)=\mu^{2} r$

with constant $\mu$ (string tension). This form is adequate for our aims because the coulombic part of the potential mainly affects the first states $(n=0)$ and becomes negligible with increasing $n^{2}$.

In order to solve Eq. (21) for any integer $n(n=$ $0,1, \ldots)$, we work in the WKB approximation [16]. The spectrum and the wavefunctions are obtained by the usual WKB procedure $[15,16]$; the spectrum is given by

$\delta_{\ell}^{(n)}=\mu \sqrt{\pi\left(2 n+\ell+\frac{3}{2}\right)} ;$

the wave function, for $r \leq r_{0}=\frac{\delta_{p}^{(n)}}{\mu^{2}}$, reads as follows:

$$
\begin{aligned}
& u_{\ell}^{(n)}(r)=\eta \sqrt{m_{Q}} \times \ell\left[\delta_{\ell}^{(n)} r-\frac{\mu^{2} r^{2}}{2}\right] \\
& \left(r \leq r_{0}\right),
\end{aligned}
$$

whereas for larger values of $r$ it decreases exponentially. Let us observe explicitly that the factor $\sqrt{m_{Q}}$ arises from the covariant normalization condition

\footnotetext{
${ }^{2}$ For example, using the Richardson potential and solving numerically the wave equation [15] one obtains a coulombic correction. to the meson mass as follows: $\Delta M_{n} / M_{n}=7.9 \%, 5.6 \%$ and $4.4 \%$ for the radial quantum number $n=0,1,2$ respectively.
} 
$\int d r\left|\Psi_{n}(r)\right|^{2}=2 M_{n}$

Let us now turn to the coupling $f_{S_{n}}=f_{1}^{(n)}$ and $f_{P_{n}}=f_{0}^{(n)}$. From the expression [17]

$f_{\ell}^{(n)}=\sqrt{\frac{3}{2}} \frac{1}{\pi M_{n, \ell}} \int_{0}^{\infty} d k k \tilde{u}_{\ell}^{(n)}(k)$,

with the function $\tilde{u}_{\ell}^{(n)}(k)$ defined as

$\tilde{u}_{\ell}^{(n)}(k)=\int_{0}^{\infty} d r \chi_{\ell}(k r) u_{\ell}(r)$,

one obtains

$f_{\ell}^{(n)}=\sqrt{\frac{3 m_{Q} \delta_{\ell}^{(n)}}{\pi}} \frac{\mu}{M_{n, \ell}}$.

Let us now reconsider the correlator Eq. (1). In the $N_{c} \rightarrow \infty$ limit the products of currents in Eq. (1) are saturated by single particle states with $J^{P}=0^{+}$and $0^{-}$, respectively. Therefore one can write

$\Pi^{\text {had }}(\epsilon)=\frac{1}{8 m_{Q}^{3}} \sum_{n=0}^{\infty}\left[\frac{\left[f_{1}^{(n)}\right]^{2}}{\epsilon+\delta_{1}^{(n)}}-\frac{\left[f_{0}^{(n)}\right]^{2}}{\epsilon+\delta_{0}^{(n)}}\right]$,

which, in the limit $m_{Q} \rightarrow \infty$, becomes

$$
\begin{gathered}
\Pi^{\text {had }}(\epsilon)=\frac{3 \mu^{2}}{8 \pi} \sum_{n=0}^{\infty}\left[\frac{\delta_{1}^{(n)}}{\epsilon+\delta_{1}^{(n)}}-\frac{\delta_{0}^{(n)}}{\epsilon+\delta_{0}^{(n)}}\right] \\
=\frac{3 \mu^{3}}{8 \sqrt{\pi}} \sum_{n=0}^{\infty} \frac{(-1)^{n+1} \sqrt{n+3 / 2}}{\epsilon+\mu \sqrt{\pi(n+3 / 2)}} .
\end{gathered}
$$

Before continuing in the analysis, a comment is in order on the model. The choice of considering only single particle states between the currents in the correlator (i.e. taking the $N_{c} \rightarrow \infty$ limit) is not too restrictive, as it can be shown by considcring, for cxample, the imaginary part of the correlator of scalar currents $\Pi_{S}$ in Eq. (1) (the same result is obtained taking $\left.\Pi_{P}\right)$. By computing the imaginary part of the quark loop diagram one obtains, for $E=-\epsilon>\rightarrow+\infty$ and at the leading order in $E$ :

$\operatorname{Im} \Pi_{S}^{\mathrm{OPE}}(E) \rightarrow \frac{3 E^{2}}{8 \pi}$.
The resonance model gives

$$
\begin{aligned}
\operatorname{Im} & \Pi_{S}^{\text {had }}=\operatorname{Im} \frac{1}{8} \sum_{n} \frac{\left[f_{1}^{(n)}\right]^{2} M_{n, 1}}{-E+\delta_{1}^{(n)}-i \epsilon} \\
& =\frac{\pi}{8} \sum_{n}\left[f_{1}^{(n)}\right]^{2} M_{n, 1} \delta\left(E-\delta_{1}^{(n)}\right) \\
& =\frac{3 \mu^{2} E}{8} \sum_{n=0}^{\infty} \delta[E-\mu \sqrt{2 \pi(n+7 / 4)}] .
\end{aligned}
$$

$\operatorname{Im} \Pi_{S}^{\text {had }}(E)$ looks very different from $\operatorname{Im} \Pi_{S}^{\text {OPE }}(E)$ in Eq. (31), due in particular to the presence of the infinite set of Dirac $\delta$ functions in Eq. (32). On the other hand it is well known that a comparison should be made only after a smearing of these distributions [2]. A convenient way to do this in the present case is make the approximation

$\sum_{n} \rightarrow \int d n$

Hence, we obtain

$$
\begin{aligned}
\operatorname{Im} & \Pi_{S}^{\mathrm{had}}=\frac{3 \mu^{2} E}{8} \int d n \delta[E-\mu \sqrt{2 \pi(n+7 / 4)}] \\
= & \frac{3 E^{2}}{8 \pi}=\operatorname{Im} \Pi_{S}^{\mathrm{OPE}}(E) .
\end{aligned}
$$

Therefore $\Pi_{S}(E)$ and $\Pi_{P}(E)$ satisfy global duality at least at the leading order for $E \rightarrow \infty$.

In the sequel we shall discuss the next-to-leading contributions that are responsible for the difference $\Pi=\Pi_{S}-\Pi_{\rho}$; for the time bcing let us comment on the value of the parameter $\mu$ and the approximate choice Eq. (22) for the potential. Assuming that the WKB approximation works reasonably well already for the the first quantum number $n=0$, we obtain

$\mu \simeq \sqrt{\frac{2}{3 \pi}}\left(m_{B}-m_{b}\right) ;$

for $m_{b}=4.6-4.7 \mathrm{GeV}$ this gives $\mu \simeq 300 \mathrm{MeV}$. The first $(n=0)$ meson state may be sensitive to the small distance, coulombic part of the interquark potential that we have omitted in Eq. (22); however, as far as we neglect consistently $\mathcal{O}\left(\alpha_{s}\right)$ terms also in the OPE counterpart of $\Pi^{\text {had }}$, this approximation is reasonable and the duality should be equally valid. 
Let us now apply the same resonance model to the correlator $\Pi(q)=\Pi_{S}(q)-\Pi_{P}(q)$ in Eq. (1). When we compute the correlator inserting hadronic states, from Eq. (30) we find ( $E=-\epsilon>0$ )

$$
\begin{aligned}
& R^{\text {had }}(E)=\operatorname{Im} \Pi^{\text {had }}(E) \\
& \quad=\frac{3 \mu^{2} E}{8} \sum_{n=0}^{\infty}(-1)^{n} \delta\left[E-\mu \sqrt{\pi\left(n+\frac{3}{2}\right)}\right] .
\end{aligned}
$$

On the other hand, from the first four terms in Eq. (7), we have

$$
\begin{aligned}
& R^{\mathrm{OPE}}(E)=\operatorname{Im} \Pi^{\mathrm{OPE}}(E) \\
& =-\frac{\pi}{4}\langle 0|\bar{q} q| 0\rangle\left[\delta(E)-\frac{m_{0}^{2}}{16} \delta^{\prime \prime}(E)\right. \\
& \left.+c_{4} \frac{m_{0}^{4}}{4 !} \delta^{(I V)}(E)-c_{6} \frac{m_{0}^{6}}{6 !} \delta^{(V I)}(E) \ldots\right] ;
\end{aligned}
$$

$c_{4}$ and $c_{6}$ are unknowns: in the model of [11] they are given by $c_{4}=\frac{5}{64}, c_{6}=\frac{61}{512}$. Also in the present casc the two expressions look very different, but they can be compared after an appropriate smearing; following [2] we consider

$$
\begin{aligned}
& \bar{R}^{\mathrm{had}}(E, \Delta)=\frac{\Delta}{\pi} \int d E^{\prime} \frac{R^{\mathrm{had}}\left(E^{\prime}\right)}{\left(E-E^{\prime}\right)^{2}+\Delta^{2}}, \\
& \bar{R}^{\mathrm{OPE}}(E, \Delta)=\frac{\Delta}{\pi} \int d E^{\prime} \frac{R^{\mathrm{OPE}}\left(E^{\prime}\right)}{\left(E-E^{\prime}\right)^{2}+\Delta^{2}} .
\end{aligned}
$$

In the limit $\Delta \rightarrow 0, \bar{R}(E, \Delta) \rightarrow R(E)$; however one has to impose $\Delta \gg \Lambda_{\mathrm{QCD}}$ [2]. As a matter of fact,

$\bar{R}(E, \Delta)=\frac{1}{2 i}[\Pi(E-i \Delta)-\Pi(E+i \Delta)]$,

and, for $\Delta \gg \Lambda_{\mathrm{QCD}}$, we are far enough from the singularities for OPE to be valid and duality to hold.

For very large $E$ the two expressions should coincide regardless of $\Delta$. From previous equations one finds that this request implies the relation

$$
\langle 0|\bar{q} q| 0\rangle=-3 \frac{0.51}{2} \frac{\mu^{3}}{\sqrt{\pi}}
$$

and, for $\langle 0|\bar{q} q| 0\rangle=(-240 \mathrm{MeV})^{3}$, this implies $\mu=$ $317 \mathrm{MeV}$; it can be noted that this number agrees nicely with the previous result Eq. (35). For smaller values of $E$, i.e. $1<E<20 \mathrm{GeV}$, the numerical

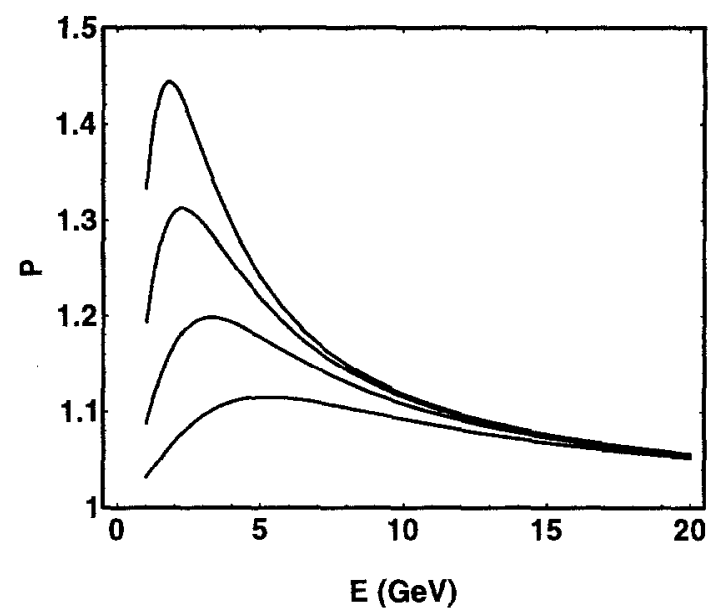

Fig. 1. Plot of the ratio (42) as a function of $E$ for several values of $\Delta$ (from top to bottom: $\Delta=1.5,2.0,3.0$ and $5.0 \mathrm{GeV}$ ).

results concerning the comparison between (38) and (39) are reported in Fig. 1, where we plot the ratio:

$P=\frac{\bar{R}^{\text {had }}}{\bar{R}^{\text {OPE }}}$

of the smeared quantities as a function of $E$ for several values of $\Delta$. We observe that, as expected, the agreement between $\bar{R}^{\text {had }}$ and $\bar{R}^{\mathrm{OPE}}$ increases with increasing $\Delta$; in particular for $\Delta=3.0 \mathrm{GeV}$ the difference does not exceed $20 \%$. In any case, for any value of the smearing parameter $\Delta$, the ratio (42) tends to unity for large $E$ values.

A further test of the soundness of our approximation is provided by the evaluation of

$\hat{F}=\sqrt{m_{b}} f_{B}$

in the limit $m_{b} \rightarrow \infty$, using the WKB formula (28) with $n=\ell=0$. We get $\hat{F}=0.26 \mathrm{GeV}^{3 / 2}$ to be compared with the QCD sum rule result, obtained without $\alpha_{s}$ corrections, i.e. in the same approximation used in this work: $\hat{F}=0.30 \pm 0.05 \mathrm{GeV}^{3 / 2}$ [18] (including $\alpha_{s}$ corrections, one would get $\hat{F}=0.41 \pm 0.04 \mathrm{GeV}^{3 / 2}$ ).

In conclusion we have tested that the resonance model we have proposed satisfies global duality: the smeared imaginary parts of the correlator, when computed by OPE or by hadron states agree with each other. When we consider local duality, however, the expressions are significantly different. As a matter of fact, starting from Eq. (30), we obtain, by the Mellin transform: 
$\tilde{\Pi}(\tau)=\frac{1}{2 \pi i} \int_{\sigma-i \infty}^{\sigma+i \infty} d \epsilon e^{\epsilon \tau} \Pi(\epsilon)$

with

$$
\begin{aligned}
\tilde{\Pi}(\tau) & =\frac{3 \mu^{3}}{8 \sqrt{\pi}} \sum_{n=0}^{\infty}(-1)^{n+1} \sqrt{\pi(n+3 / 2)} \\
\times e^{-\tau \mu \sqrt{\pi(n+3 / 2)}} &
\end{aligned}
$$

$\tilde{\Pi}$ can be expanded for small $\tau$; we find numerically

$\tilde{\Pi}(\tau)=\frac{3 \mu^{3}}{8 \sqrt{\pi}}\left[-0.51+\frac{\mu \sqrt{\pi} \tau}{2}-0.46 \frac{\mu^{2} \pi \tau^{2}}{2} \ldots\right]$.

Since

$\Pi(\epsilon)=\int_{0}^{\infty} d \tau e^{-\tau \epsilon} \tilde{\Pi}(\tau)$,

we find

$\Pi^{\text {had }}(\epsilon)=-3 \frac{0.51 \mu^{3}}{8 \sqrt{\pi}} \frac{1}{\epsilon}\left[1-\frac{\tilde{m}_{0}}{\epsilon}+0.93 \frac{\tilde{m}_{0}^{2}}{\epsilon^{2}} \ldots\right]$.

The factor multiplying $\frac{1}{\epsilon}$ coincides numerically with $\frac{\langle 0| \bar{q} q[0\rangle}{4}$ for $\mu=317 \mathrm{MeV}$ (see Eq. (41)). As for the mass parameter $\tilde{m}_{0}$, numerically we find $\tilde{m}_{0}=560$ $\mathrm{MeV}$. In conclusion we get $(\epsilon<0)$ :

$\Pi^{\mathrm{had}}(\epsilon)=\frac{\langle 0|\bar{q} q| 0\rangle}{4 \epsilon}\left[1-\frac{\tilde{m}_{0}}{\epsilon}+0.93 \frac{\tilde{m}_{0}^{2}}{\epsilon^{2}}+\ldots\right]$.

A comparison between Eq. (7) and Eq. (48) shows a violation of local duality which manifests itself in the form of an unexpected term in the $\frac{\hat{s}}{\epsilon}$ asymptotic expansion.

\section{Conclusions}

Studying simple correlators of quark currents, we have found, in a particular quark model and in the $N_{c} \rightarrow \infty$ limit, an explicit example of violation of local duality which occurs in a condition where global duality is verified. The difference between the hadronic and the OPE spectral functions is made evident by the $\Lambda / \epsilon$ term which is absent in the expansion predicted by OPE.

The calculation in QCD of correlators of quark operators between hadronic states, as required for the evaluation of, e.g., the $B_{d}$ and $\Lambda_{b}$ inclusive decay widths, is, of course, beyond the aims of the present analysis. However, the example described in this paper supports the suggestion that the $\Lambda_{b}$ lifetime data could find an explanation in the presence of a $1 / m_{Q}$ correction not included in the usual QCD-OPE expansion.

\section{Acknowledgements}

We thank N. Paver for useful discussions. The work of C.A.D. has been supported in part by the FRD (South Africa).

\section{References}

[1] J. Chay, H. Georgi and B. Grinstein, Phys. Lett. B 247 (1990) 399 ;

I. Bigi, M. Shifman, N. Uraltsev and A.I. Vainshtein, Phys. Rev. Lett. 71 (1993) 496;

B. Blok, L. Koyrakh, M. Shifman and A.I. Vainshtein, Phys. Rev. D 49 (1994) 3356;

A.V. Manohar and M.B. Wise, Phys. Rev. D 49 (1994) 1310; A.F. Falk, M. I uke and M.J. Savage, Phys. Rev. D 523 (1996) 2491.

[2] E.C. Poggio, H.R. Quinn and S. Weinberg, Phys. Rev. D 13 (1976) 1958.

[3] C.G. Boyd, B. Grinstein and A.V. Manohar, Phys. Rev. D 54 (1996) 2081.

[4] M.B. Volushin and M. Shifman, Yad. Fiz. 47 (1988) 801 (Sov. Journ. Nucl. Phys, 47 (1988) 511).

[5] G. Altarelli, G. Martinelli, S. Petrarca and F. Rapuano, Phys. Lett. B 382 (1996) 409.

[6] M. Neubert and C.T. Sachrajda, Nucl. Phys. B 483 (1997) 339.

[7] P. Colangelo and F. De Fazio, Phys. Lett. B 387 (1996) 371.

[8] I.I. Bigi et al., in: B decays, S. Stone ed., 2nd edition (World Scientific, Singapore, 1994) p. 132.

[9] J. Richman, talk given at the 28th International Conference on High Energy Physics, ICHEP96, Warsaw, Poland (25-31 July 1996), to appear in the Proceedings.

[10] A. Deandrea, N. Di Bartolomeo, R. Gatto and G. Nardulli, Phys. Lett. B 398 (1997) 353.

[11] M. Shifman, Theory of Preasymptotic Effects in Weak Inclusive Decays, in: Proc. of the Workshop Continuous Advances in QCD, ed. A. Smilga (World Scientific, Singapore, 1994) p. 249;

M. Shifman, in: Proc. of the Johns Hopkins and Pascos 
Workshop: Particle, Strings and Cosmology, ed. J. Bagger (World Scientific, Singapore, 1996).

[12] B. Chibisov, R. David Dikeman, M. Shifman and N.G. Uraltsev, Int. J. Mod. Phys. A 12 (1997) 2075.

[13] S.V. Mikhailov and A.V. Radyushkin, JETP Lett. 43 (1986) 712; Sov. J. Nucl. Phys. 49 (1989) 494

[14] M. Abramowitz and I.A. Stegun, Handbook of Mathematical Functions, New York (1965).
[15] P. Colangelo, G. Nardulli and M. Pietroni, Phys. Rev. D 43 (1991) 3002.

[16] P. Cea, P. Colangelo, G. Nardulli, G. Paiano and G. Preparata, Phys. Rev. D 26 (1982) 1157.

[17] P. Cea and G. Nardulli, Phys. Rev. D 34 (1986) 1863.

[18] M. Neubert, Phys. Rev. D 45 (1992) 2451; D 46 (1992) 1076. 